\title{
Marty Seligman: "Das pathologische Krankheitsmodell hat uns bei ganz normalen Leuten nicht weitergeholfen»
}

\author{
Ein Interview über positive Gesundheit
}

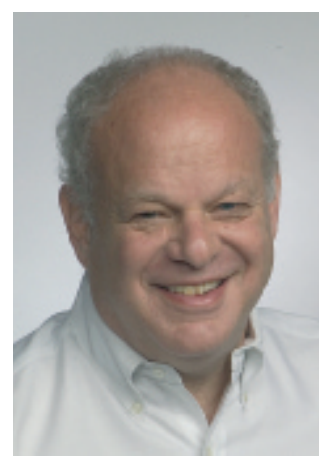

Prof. Dr. Martin E.P. Seligman ist Professor für Klinische Psychologie und Leiter des Instituts für Positive Psychologie an der University of Pennsylvania, PA. Neben seinen bahnbrechenden Arbeiten zur erlernten Hilflosigkeit, hat Prof. Seligman das Feld der Positiven Psychologie begründet, über 170 wissenschaftliche Arbeiten in hochrangigen Journals veröffentlicht und ist Autor von über 20 Bestsellern ("Learned Optimism», "What You Can Change and What You Can't», "The Optimistic Child", "Abnormal Psychology»). Prof. Seligman war Präsident der Abteilung für Klinische Psychologie der Amerikanischen Gesellschaft für Psychologie und hat zahlreiche internationale Auszeichnungen erhalten (u.a.: "Distinguished Scientific Contribution Award" der American Psychological Association, "William James Fellow Award" für Beiträge zur Grundlagenforschung und "James McKeen Cattell Fellow Award" für die Anwendung psychologischen Wissens).

Das Gespräch mit ihm fand im Rahmen eines regelmäßigen Treffens des Steering Commitees der Forschergruppe zu Positive Health am Institut für Positive Psychologie der University of Pennsylvania statt. Das Interview führte Dr. Yvonne Nestoriuc, Beraterin im Projekt "Super Healthy" der Positive-Health-Forschergruppe und akademische Rätin am Lehrstuhl für Klinische Psychologie und Psychotherapie der Philipps-Universität Marburg.

Lieber Marty Seligman, vielen Dank für Ihre Bereitschaft, an diesem Interview teilzunehmen. Sie beschäftigen sich bereits seit vielen Jahren mit positiven menschlichen Erfahrungen, mit positiven Charaktereigenschaften sowie der Möglichkeit, das individuelle menschliche Leben glücklicher und erfüllter zu gestalten. Wie sehen Sie das Verhältnis der positiven Psychologie zur klinischen Psychologie?

Marty Seligman: Im Jahr 2000 habe ich für unser Zentrum «Positive Psychologie» eine Leitlinie entworfen, die meine Vision der positiven Psychologie sehr gut widergibt: 'Our core goal is to foster the scientific investigation of positive subjective experience, of positive individual traits and of positive institutions. When a nation is in surplus, at peace, and not in social turmoil, the sciences it supports turn from being only about defence and damage to being about achieving what is best within us. This is the vision that inspires psychology now and will create the science of tomorrow.' Ich verstehe die positive Psychologie in vielerlei Hinsicht als sinnvolle Ergänzung zur klinischen Psychologie. Sie bietet Strategien, die uns helfen können, mehr positive Emotionalität in unser Leben zu bringen. Insbesondere wenn wir an Erkrankungen wie die Depression denken, deren Symptomatik so eng verwandt ist mit den Konstrukten der positiven Psychologie (vgl. Optimismus, Kohärenzsinn, Vertrauen, Hoffnung, Lebenssinn, persönliche Stärken - Anmerkung der Interviewerin), liegt es nahe, mit den Klienten neben dem Abbau der belastenden Faktoren vor allem auch den Aufbau positiver Erlebniswelten und Lebensfacetten zu fördern - ich spreche lieber von Klienten als von Patienten. In diesem Sinne kann die positive Psychologie das Leben normaler Menschen ebenso wie das von Klienten bereichern und verbessern.

\section{KARGER}

Fax +49 7614520714

Information@Karger.de

www.karger.com (c) 2009 S. Karger GmbH, Freiburg

Accessible online at:

www.karger.com/ver
Dr. Yvonne Nestoriuc

Philipps-Universität Marburg

Klinische Psychologie und Psychotherapie

Gutenbergstraße 18, 35032 Marburg, Deutschland

Tel. +49 64212824051

yn@staff.uni-marburg.de 
Bislang sind eine Vielzahl von Konstrukten in der positiven Psychologie entwickelt, operationalisiert und untersucht worden. Zu den bekanntesten zählen sicherlich Glück, Optimismus, Flow, Lebenssinn und Liebe. Für welches Konstrukt sehen Sie die meisten Anwendungsmöglichkeiten in der Klinischen Psychologie und Psychotherapie?

Marty Seligman: Da habe ich zwei klare Favoriten: Hoffnung und Optimismus; aus dem einfachen Grund, dass sie erlernbar sind. Wir konnten zeigen, dass sich positive Emotionen wie Hoffnung und ein positiver Blick auf die Zukunft mit simplen Interventionen wie zum Beispiel dem Dankbarkeitstagebuch erfolgreich trainieren lassen. Dazu muss gesagt werden, dass auch hier keine Wunder geschehen. Neben einem erheblichen Erblichkeitsfaktor von rund $50 \%$ für positive Emotionalität und Optimismus ist der Anteil des erlernbaren Optimismus deutlich geringer. Wir können einen Griesgram also nicht in einen Sonnenschein verwandeln. Nichtsdestotrotz eröffnet das erfolgreiche Training individueller Optimismusanteile vielfältige Anwendungsmöglichkeiten insbesondere für die Prävention von psychischen und körperlichen Erkrankungen.

Analog zur Definition positiver Psychologie haben Sie im letzten Jahr mit einem Artikel in der Zeitschrift «Applied Psychology» das Forschungsfeld der Positiven Gesundheit entworfen. Im Fokus steht dabei die Überzeugung, dass die wissenschaftliche und gesamtgesellschaftliche Beschäftigung mit Gesundheit anstatt Krankheit über kurz oder lang zu einer höheren Lebenserwartung und Kostenersparnissen im Gesundheitssektor führen wird. Angeregt durch diese Hypothese, haben sich bereits in den letzten knapp 2 Jahren einige Forschungsansätze im Rahmen der Positiven Gesundheit entwickelt. Was zeichnet das Forschungsgebiet der Positiven Gesundheit aus und welche Ergebnisse halten Sie aktuell für besonders spannend?

Marty Seligman: Wir wissen heute, dass die pathologische Krankheitslehre nicht sonderlich hilfreich war und ist, wenn es um den Umgang mit ganz normalen Leuten geht. Mit dem pathologischen Modell gelingt es weder, normale Gesundheitsentwicklung vorherzusagen, noch, Faktoren positiver Gesundheit zu untersuchen. Das Feld der Positiven Gesundheit ist noch sehr jung. Auch wenn die Beschäftigung mit dem Thema Gesundheit so alt ist wie die Menschheit selbst und an einigen Universitäten Gesundheitspsychologie bereits als Fach gelehrt wird, so ist doch der Blickwinkel heute völlig neu. Einige der derzeit interessantesten Ergebnisse zeigen, dass Hoffnung und Optimismus als Schutzfaktoren gegen Herz-Kreislauf-Erkrankungen wirken. In Längsschnittuntersuchungen konnte Laura Kubzansky von der Harvard School of Public Health zeigen, dass über einen Zeitraum von 10 Jahren das Risiko koronarer Herzerkrankungen sowie tödlicher Herzinfarkte bei Personen mit ausgeprägter positiver Emotionalität (wie z.B. Hoffnung) und hohem Optimismus deut- lich geringer war, während sich hohe Werte auf Depressionsskalen deutlich negativ auf das Risiko kardiovaskulärer Erkrankungen auswirkten. Ebenso spannend ist die Frage nach den vermittelnden Faktoren beim Zusammenhang zwischen hohem Bildungsniveau und guter Gesundheit, der sich in vielen unterschiedlichen Untersuchungen immer wieder gezeigt hat. Erklärungsansätze der positiven Psychologie postulieren Zukunftsorientierung als eine wichtige Eigenschaft, die den Effekt von Bildung auf Gesundheit aufklären kann. Dies lässt sich wiederum mit dem Ergebnis in Zusammenhang bringen, dass sich Menschen, die mehr Erziehung und Bildung genossen haben, in der Regel auch als optimistischer beschreiben. Aktuell wird im Bereich der Kinder- und Jugendpsychologie unter anderem der Frage nachgegangen, ob Zukunftsorientierung oder die Fähigkeit zum Belohnungsaufschub der Motor dieses Zusammenhangs sind.

Welches sind die Faktoren, die Ihrer Meinung nach am besten geeignet sind, positive Gesundheit zu beschreiben?

Marty Seligman: Genau diese Frage zu beantworten, ist die Hauptaufgabe des Komitees zur Untersuchung positiver Gesundheit, das ich mit Unterstützung der Robert Wood Johnson Foundation hier zusammengerufen habe. Dabei kommen verschiedene Forschungsansätze aus der Klinischen, Gesundheits-, Persönlichkeitspsychologie, Psychiatrie, Entwicklungssowie Kinder- und Jugendpsychologie zum Einsatz. Generell beziehen wir in unsere Forschung die ganze Palette von Variablen subjektiven Erlebens, funktionaler Gesundheit und gezeigten Verhaltensweisen ein. Zu meinen aktuellen Favoriten zählen sicherlich Lebenszufriedenheit und Optimismus.

Bereits Mitte der 1940er Jahre hat die Weltgesundheitsorganisation Gesundheit definiert als «mehr als nur die Abwesenheit von Krankheit». Betrachten Sie Gesundheit als bipolares Konstrukt oder sollten wir uns positive Gesundheit und Krankheit vielmehr als zwei weitgehend unabhängige Dimensionen vorstellen?

Marty Seligman: In unseren Untersuchungen finden wir regelmäßig eine Korrelation von $r=-0,40$ zwischen positiven Gesundheitsvariablen und physischer Krankheit. Dieser negative Zusammenhang von moderater Ausprägung spricht dafür, positive Gesundheit und Krankheit als zwei unterschiedliche Dimensionen zu betrachten, die jedoch nicht völlig unabhängig voneinander sind. So ist im Schnitt einerseits ein gewisses Maß an Freiheit von körperlichen Erkrankungen notwendig für ein positives Gesundheitserleben. Andererseits ist das individuelle positive Gesundheitserleben zu einem mindestens ebenso großen Anteil unabhängig von physischen Faktoren. Mit diesem Model kann somit sowohl Steven Hawking dargestellt werden, der ein relativ hohes Ausmaß an physischer Krankheit und ein ebenso hohes Ausmaß an positiver Gesundheit aufweist, als auch der hy- 
pochondrische Patient, der im Gegenteil wenig physische Krankheit und deutliche Einschränkungen im positiven Gesundheitserleben aufweist.

Der Prototyp für extrem positive Werte auf beiden Dimensionen, also minimale körperliche Krankheit bei maximaler positiver Gesundheit, sind die Supergesunden («Super Healthy People»). Zurzeit untersuchen wir mit einer parallel von Dr. Arthur Barsky an der Harvard Medical School in Boston durchgeführten Pilotstudie das Konzept Supergesundheit. Über Annoncen suchen wir nach Menschen, die nie krank werden, sich supergesund fühlen und ihr ganzes Leben lang weder in der Schule noch bei der Arbeit Fehlzeiten durch Krankheiten angehäuft haben. Was denken Sie, könnten wir von solchen supergesunden Menschen lernen?

Marty Seligman: Die Supergesunden sind ein besonders interessantes Forschungsthema. Wenn wir uns die Verteilung von positiver Gesundheit als eine Gauß'sche Normalverteilungskurve vorstellen, sprechen wir hier über die besten $5 \%$ dieser Verteilung. Und es geht dabei nicht um spezifische Ausnahmen wie über Hundertjährige, die Marathon laufen, sondern um die Art von positiver Gesundheit, die wir uns alle wünschen. Eine optimale Gesundheit bis ins hohe Alter, die es uns ermöglicht, unserer Arbeit mit Freude nachzugehen, Hobbys und soziale Kontakte zu verwirklichen und körperlich aktiv zu sein. Interessant sind dabei natürlich auch interkulturelle Vergleiche, wobei die Unterschiede im Umgang mit Gesundheit zwischen den USA und Deutschland natürlich deutlich weniger drastisch ausfallen werden als zum Beispiel jene zwischen westlichen und östlichen Kulturen. Besonders interessieren uns die psychologischen Charakteristika dieser
Personen. Sind supergesunde Menschen hoffnungsfroher und optimistischer als andere? Haben sie mehr oder erfüllendere soziale Kontakte, zeigen sie mehr positives Engagement an der Arbeit, erleben sie mehr Flow und generell mehr positive Emotionalität? Mit ersten Antworten auf diese Fragen, könnten wir Variablen definieren, die förderlich sind für positive Gesundheit, und deren Einfluss auf die Lebenserwartung im Längsschnitt untersuchen. Von weitreichendem Interesse sind natürlich Faktoren, die zu lebenslanger ausgezeichneter Gesundheit beitragen und erlernbar oder trainierbar sind. Variablen wie soziale Kompetenz und Beziehungen, Optimismus und Hoffnung könnten wir anschließend mit psychologischen Mitteln trainieren und in die Gesundheitsprophylaxe einbauen.

Wo sehen Sie die Zukunft der Forschung zur positiven Gesundheit?

Marty Seligman: Die Forschung zur positiven Gesundheit knüpft besonders an das Feld der Verhaltensmedizin an. Hier sehe ich die Zukunft der Forschungsstränge vor allem in der Prävention von körperlichen und psychischen Krankheiten. Dementsprechend sehe ich eine der Hauptaufgaben der Forschung zur positiven Gesundheit darin, Hypothesen und neue Ansätze für die Prävention von Krankheiten zu generieren.

\section{Vielen Dank für das Gespräch!}

Weitere Informationen zu Konstrukten der positiven Psychologie und positiven Interventionen (mit der Möglichkeit, selbst Fragebögen auszufüllen) erhalten Sie unter: www.authentichappiness.sas.upenn.edu. Informationen zum Superhealthy-Projekt erhalten Sie unter: www.uni-marburg. de/supergesund. 